Itinéraires Itinéraires

Littérature, textes, cultures

2018-2 et $3 \mid 2019$

Les imaginaires de la traduction

\title{
D'un imaginaire colonial à un autre : Ferdinand Oyono en traduction allemande
}

From One Colonial Imaginary to Another. Ferdinand Oyono in German

Translation

El-Shaddai Deva

\section{OpenEdition}

\section{Journals}

Édition électronique

URL : http://journals.openedition.org/itineraires/4817

DOI : $10.4000 /$ itineraires.4817

ISSN : 2427-920X

Éditeur

Pléiade

Référence électronique

El-Shaddai Deva, «D'un imaginaire colonial à un autre : Ferdinand Oyono en traduction allemande », Itinéraires [En ligne], 2018-2 et 3 | 2019, mis en ligne le 20 février 2019, consulté le 17 juin 2019. URL http://journals.openedition.org/itineraires/4817 ; DOI : 10.4000/itineraires.4817

Ce document a été généré automatiquement le 17 juin 2019.

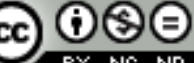

Itinéraires est mis à disposition selon les termes de la licence Creative Commons Attribution - Pas d'Utilisation Commerciale - Pas de Modification 4.0 International. 


\section{D'un imaginaire colonial à un autre : Ferdinand Oyono en traduction allemande}

From One Colonial Imaginary to Another. Ferdinand Oyono in German

Translation

El-Shaddai Deva

1 Dans son article «Schwarze Haut, weiße Sprachen », Doris Kolesh part du constat de la mise en contact de plusieurs langues dans le roman Une vie de boy de l'écrivain camerounais Ferdinand Oyono (le français, langue de la narration, y est parsemé d'expressions d'exclamation en langue espagnole, de termes en langue ewondo ainsi que de formes mixtes créées à partir de la combinaison de plusieurs langues) pour montrer comment cette configuration lui permet de déconstruire le discours colonial (1992: 17). Les langues mises en scène apparaîtraient selon Kolesh comme des «mode[s] de communication de valeur égale ${ }^{1} »(19)$, ce qui permettrait de mettre en question l'autorité de la langue coloniale française. Il est intéressant de noter que cette co-présence de diverses langues se lit également dans Le Vieux Nègre et la Médaille, un autre roman d'Oyono publié la même année que le précédent, où le français est mis en relation avec l'anglais et le mvema, une langue locale camerounaise. L'écriture de Ferdinand Oyono procède donc d'un imaginaire linguistique ${ }^{2}$ pluriel. Elle ne s'opère pas dans les limites d'une seule langue, mais conçoit sa langue comme une langue parmi d'autres, la met en relation avec d'autres, la fait apparaître dans son rapport à d'autres. En étudiant l'imaginaire linguistique et poétique des œuvres de Ferdinand Oyono, nous analysons la manière dont les traductions respectives desdites œuvres en allemand reproduisent cet imaginaire ${ }^{3}$. Partant de la différence des langues française et allemande, et de la différence entre les imaginaires coloniaux français et allemand, nous nous intéressons à quelques disparités entre les originaux et les traductions, notamment au niveau de la traduction du français petit-nègre et de la configuration de l'oralité. Il ne s'agit pas de vérifier si les traductions sont fidèles ou infidèles, bonnes ou mauvaises, mais plutôt de déterminer s'il existe, dans la pratique des traducteurs, une certaine continuité et une 
certaine cohérence dans leur traitement de l'imaginaire des textes originaux. Bien évidemment, l'article se garde d'opérer une critique normative des traductions et de juger les traducteurs. En réalité, comme nous le rappelle Susan Bassnett,

La traduction ne se passe jamais dans un vide; elle se réalise toujours dans une continuité; et le contexte dans lequel est produite la traduction affecte nécessairement le comment de la traduction. Tout comme les normes et contraintes de la culture source jouent leur rôle dans la création du texte orignal, les normes et conventions de la culture cible jouent tout de même un rôle dans la création de la traduction $^{4}$. (Bassnett et Lefevere 2010 : 93)

2 La traduction est un processus extrêmement complexe qui implique une chaîne d'agents dont le traducteur n'est qu'un simple maillon (Milton et Bandia $2009: 1$ ).

\section{L'imaginaire linguistique de l'œuvre d'Oyono}

Édouard Glissant présente, notamment dans Introduction à une poétique du divers (1996), les imaginaires monolingue et multilingue comme deux types de rapport du sujet à sa langue et aux autres langues. L'imaginaire monolingue ${ }^{5}$ est, selon lui, à la base des littératures des communautés dites ataviques, celles « qui sont basées sur l'idée d'une Genèse, c'est-àdire d'une création du monde, et sur l'idée d'une filiation de la communauté à cette Genèse » (34). Les grands textes fondateurs de ces communautés seraient l'expression d'un « cri poétique », celui d'une communauté « non encore sûre de son identité » et qui a besoin de «se rassurer face à une menace» (36). Le sujet monolingue se représente sa langue comme une langue sacrée, la langue de sa communauté, inspirée par le dieu de sa communauté. " $\mathrm{Ce}$ n'est pas la langue du dieu ou des autres communautés et, littérairement, la langue va acquérir une fonction d'absolu et de sacralisation dont on verra les conséquences jusqu'aujourd'hui » (ibid.).

4 L'auteur monolingue est celui qui écrit dans sa langue sans tenir compte des autres langues, qui a un imaginaire de sa propre langue comme d'une langue propre, sacrée, sacralisée, qu'il importe de défendre, d'illustrer et de préserver. L'imaginaire monolingue confond le paradigme de la propriété avec celui de la propreté de la langue (Auzas 2009: $41)$ : il se représente sa propre langue, la langue de sa communauté et de son territoire comme une langue propre, pure et inspirée, qu'il conviendrait de protéger de toute contamination par une autre langue. Il nourrit, dans ses rapports aux autres langues (ou aux langues des autres), une attitude de réserve, de silence, de renfermement et d'exclusion (voir Glissant 1996 : 42).

5 L'imaginaire linguistique des romans de Ferdinand Oyono est tout le contraire de l'imaginaire monolingue. Comme noté dans l'introduction, le français, langue d'écriture dans Le Vieux Nègre et la Médaille ${ }^{6}$ et Une vie de boy ${ }^{7}$, est mis en relation avec d'autres langues : l'espagnol, ou plutôt « un baragouin d'espagnol et de pahouin mêlés » (VB: 9), l'anglais, par la présence dans le texte de quelques mots anglais dont certains sont expliqués en notes de bas de page, comme stock-fish (VNM : 47), africa-gin (VNM : 20), drill (VB : 28), mais surtout des langues locales camerounaises (ndjem et ewondo dans Une vie de boy, et mvema dans Le Vieux Nègre et la médaille). L'écriture d'Oyono conçoit sa langue de narration (le français), comme une langue entre autres et la met en relation avec d'autres langues. Comme chez tous les auteurs africains francophones, son écriture est basée sur un imaginaire multilingue. Bien qu'écrivant en langue française, il ne la sacralise pas et ne voit pas en elle une langue de Dieu, de son dieu ou de sa communauté. Il 
est au contraire conscient de la réalité que toute langue ne peut exister et se pratiquer que dans sa relation avec d'autres langues. Sa pratique du français est donc une pratique multilingue : elle tient compte de l'existence d'autres idiomes, qui s'expriment d'une manière ou d'une autre dans son écriture ${ }^{8}$.

\section{Le rapport entre les langues chez Oyono}

6 Si la présence de l'anglais et de l'espagnol dans le texte se limite à quelques emprunts lexicaux, le ndjem et l'ewondo d'une part, et le mvema d'autre part, sont respectivement présentes dans l'hypo-structure des deux textes. Dans Le Vieux Nègre et la Médaille, la langue mvema est présente tout au long du texte, tant au niveau du discours qu'au niveau de l'histoire. Ainsi, on notera que la plupart des personnages africains sont monolingues, quoique leurs discours soient rapportés en langue française. Une bonne partie des personnages locaux, en effet, ne parlent que le mvema. C'est le cas du personnage principal, le vieux Meka, dont la compétence linguistique en français se limite à «oui oui » (114). C'est aussi le cas des chefs et dignitaires locaux invités à la réception au Foyer Africain à l'occasion de la visite du Haut-Commissaire. La communication entre les personnages noirs et blancs n'est possible que par l'intermédiaire d'un interprète (133-137). Les discours des personnages noirs, rapportés dans le roman en langue française, ont donc manifestement été traduits du mvema. L'auteur n'a pas écrit de manière monolingue, mais en tenant compte d'une autre langue, d'autres langues. Bien qu'il écrive dans un français correct et standard, il ne le défend pas et ne l'illustre pas. Bien au contraire, il le force à être en relation avec la langue mvema. Et là même où le texte parle français, il laisse parler en arrière-plan une autre langue.

Dans Une vie de boy, on peut distinguer deux niveaux, donc deux instances de narration. Dans une sorte de prologue, le premier narrateur relate les circonstances dans lesquelles il fait la connaissance du jeune mourant Toundi Ondoua, dont il reçoit, en deux volumes, le journal intime en langue ewondo: «C'est ainsi que je connus le journal de Toundi. Il était écrit en ewondo, l'une des langues les plus parlées au Cameroun. Je me suis efforcé d'en rendre la richesse sans trahir le récit dans la traduction que j'en fis et qu'on va lire " (VB : 14). Le second niveau de narration, où se déroule l'histoire relatée par le personnage principal Toundi, est ainsi présenté comme une traduction. Il est néanmoins intéressant de constater que si le journal est écrit en ewondo, la plupart des personnages du texte parlent plutôt le ndjem. Toundi, le narrateur de sa propre histoire, est donc en même temps un traducteur, rendant en ewondo le discours non seulement des monolingues francophones, mais aussi des monolingues ndjemophones. Dans cette situation, la langue française n'est pas - du moins dans le journal de Toundi - l'hyper-langue, mais l'une des deux hypolangues du texte ewondophone. Elle est mise en rapport avec d'autres langues.

Ainsi, le paysage linguistique des deux romans est composé de deux groupes de monolingues (francophones et afrophones) et de deux groupes de multilingues (européens et africains). Les monolingues francophones sont généralement des personnages européens : les Commandants, le Haut-Commissaire, les commissaires de police, le régisseur de prison, les ingénieurs, médecins, vétérinaires et instituteurs européens travaillant pour le compte de l'administration coloniale, etc. Ces locuteurs du français ne parlent pas les langues camerounaises, à l'exception notable du commandant dans Une vie de boy, qui montre une connaissance passive du ndjem (VB : 148-149). Leur monolinguisme se justifie par une aversion et un mépris vis-à-vis de la langue des 
indigènes, considérée comme sans valeur particulière et comme sous-langue. De l'autre côté, les monolingues njemophones ou mvemophones ne parlent pas le français, parce qu'ils n'ont eu la chance ni d'aller à l'école ni de travailler dans les maisons et bureaux des colons. On notera la présence de vendeurs grecs et de missionnaires américains, dont on peut penser qu'ils parlent aussi le grec ou l'anglais, respectivement.

Entre les communautés des monolingues se trouvent les personnages multilingues, parlant parfaitement leur langue première et ayant des connaissances au moins rudimentaires de l'autre langue. C'est ainsi qu'on peut distinguer entre les multilingues européens que sont les membres du clergé dans les deux romans ${ }^{9}$, et d'autre part les multilingues africains que sont les personnages exerçant des tâches subalternes. C'est parmi ces personnages multilingues, qui sont en contact permanent avec les deux mondes (celui des colons et celui des colonisés), que l'on compte les locuteurs du parler petitnègre.

\section{Le petit-nègre}

10 Le petit-nègre est une sorte de « langue auxiliaire artificielle » et "d'espéranto à usage colonial » (Van den Avenne 2005 : 130) qu'Oyou Sénamin Amedegnato définit « comme un français incorrect, sommaire et rudimentaire parlé par les indigènes des anciennes colonies françaises " (Amdegnato $2010:$ 87). Dans son ouvrage Vocabulaire comparatif de plus de soixante langues ou dialectes parlés à la Côte d'Ivoire, Maurice Delafosse consacre quelques pages à ce parler utilisé "par nos tirailleurs et nos employés et domestiques indigènes" (Delafosse 1904: 263) qu'il décrit comme "la simplification naturelle et rationnelle de notre langue si compliquée » (ibid.). Le petit-nègre serait le résultat logique de l'incapacité du colonisé à assimiler une langue aussi complexe que le français :

Comment voudrait-on qu'un Noir, dont la langue est d'une simplicité rudimentaire et d'une logique presque toujours absolue, s'assimile rapidement un idiome aussi raffiné et illogique que le nôtre ? C'est bel et bien le Noir - ou, d'une manière plus générale, le primitif - qui a forgé le petit-nègre, en adaptant le français à son état d'esprit. (Delafosse 1904 : 264)

Comme le développe Cécile Van den Avenne, le petit-nègre est aussi appelé «françaistirailleur » du fait qu'il a «servi de langue véhiculaire au sein de l'armée coloniale en Afrique de l'Ouest, pour la communication entre officiers blancs et tirailleurs noirs, mais également pour la communication entre tirailleurs de groupes ethnolinguistiques différents» (Van den Avenne 2005: 11). Il n'était donc pas un registre exclusif des « indigènes non civilisés ", mais servait de sabir entre colons et colonisés. C'est d'ailleurs ce que soutient Maurice Delafosse qui, en tant qu'administrateur colonial, recommandait l'utilisation du petit-nègre comme langue de communication avec les indigènes : «si nous voulons nous faire comprendre vite et bien, il nous faut parler aux Noirs en nous mettant à leur portée, c'est-à-dire leur parler petit-nègre » (Delafosse $1904: 264)$. Van den Avenne soutient "que l'armée coloniale française a contribué à construire et à fixer » le petitnègre (2005 : 11-12). C'est ainsi que fut publié, en 1916, un manuel intitulé Le français tel que le parlent nos tirailleurs sénégalais à l'intention des gradés français, afin de leur permettre de «se faire comprendre en peu de temps, de leurs hommes, de donner à leurs théories une forme intelligible pour tous et d'intensifier ainsi la marche de l'instruction " (ibid. : 128). Bien que plus tard, l'administration coloniale ait privilégié l'enseignement du 
français standard, il n'en demeure pas moins que, comme l'écrit Van den Avenne, les colonisés ont continué de se servir du petit-nègre dans les conversations quotidiennes :

Mais, finalement, il semblerait que ce soit l'approche universaliste qui ait prévalu au sein de la hiérarchie militaire, s'illustrant linguistiquement par le choix d'utiliser et d'enseigner le français standard, contre l'usage des diverses langues indigènes et contre l'usage du petit-nègre. Cependant, cela ne veut pas dire que cette approche culturaliste n'ait pas marqué pour longtemps les pratiques au sein des troupes. Des témoignages tardifs rendent compte de la durabilité de l'usage du « sabir ». (Van den Avenne $2005:$ 136)

Les occurrences du parler petit-nègre dans les romans de Ferdinand Oyono rendent ainsi compte de l'imaginaire linguistique dans les colonies françaises. Elles montrent comment le contexte colonial a permis aux colons et aux colonisés de créer, ensemble, un registre de communication.

\section{Du parler petit-nègre en allemand ?}

$13 \mathrm{Au}$ vu de ce qui précède, il est évident que les textes de Ferdinand Oyono posent un sérieux défi au traducteur allemand, notamment là où ce dernier est confronté aux énoncés en petit-nègre. À l'inverse du français en effet, il n'y a pas eu, dans l'histoire coloniale allemande, une politique visant à répandre l'apprentissage de l'allemand à tous les colons. Comme l'écrit Lasme Elvis Bedi, l'administration coloniale allemande n'a pas montré le même enthousiasme pour la promotion de la langue allemande dans ses colonies qu'ont montré les puissances britanniques et françaises dans leurs colonies respectives. Les raisons en seraient «la configuration linguistique complexe dans les colonies, la difficulté quant à l'envoi d'enseignants à partir de la métropole, et l'attitude de l'administration coloniale vis-à-vis du principe de l'éducation de masses. Le dernier semblerait être la plus importante »(Bedi $2006: 74)^{10}$.

Étant donné que l'allemand n'a pas été vulgarisé, que l'administration allemande n'a pas eu à recruter autant de tirailleurs que la France et que l'allemand n'a été que brièvement langue officielle dans quelques colonies d'Afrique, il ne s'est pas constitué un parler de l'allemand qui correspondrait au français petit-nègre ${ }^{11}$.

Il va donc de soi que les traductions allemandes des textes de Ferdinand Oyono ne rendent pas toujours justice à la structure hybride du petit-nègre. À certains endroits, les versions allemandes donnent l'impression de traductions de textes écrits en français monolingue contenant quelques exotismes africains. Comme nous le voyons dans les exemples suivants, la traduction manque de continuité dans sa manière de résoudre le problème que pose le parler petit-nègre de différentes manières ${ }^{12}$ :

- C'est le vin d'honneu', mon commandant, dit-il avec un large sourire, le vin d'honneu' seulement.

[...] En rangs par deux, quinze indigènes, accablés par de volumineuses caisses, titubaient au pied de l'escalier. Le noir qui avait frappé à la porte désigna avec son casque au bout de la main les trois premiers.

- Sampagne! dit-il.

Il désigna les trois suivants.

- Sampagne, répéta-t-il.

Puis le septième :

- Pas tout à fait sampagne, dit-il, mais même chose, $f c h f c h f c h f c h f c h . .$. fit-il. (VNM : 64-65). 
„Der Festwein, mein Kommandant“, sagte er mit einem breitem Lächeln, „nur der Festwein".

[...] Am Fuß der Treppe schwankten vierzehn Eingeborene in Zweierreihen, gebeugt unter der last großer Kisten, heran. Der Schwarze, der an die Tür geklopft hatte, zeigte mit dem Helm in der ausgestreckten Hand auf die drei ersten Reihen.

„Sampanjer!“ sagte er

Er wies auf die drei folgenden Reihen und wiederholte :

„Sampanjer!“

Und schließlich auf die siebte zeigend, sagte er :

„Nicht richtig Sampanjer, aber so ähnlich Fschfschfschfschfsch“ (DaNM : 67)

On peut concéder que dans ces passages, la traduction rend justice, dans une certaine mesure, à la tonalité du langage du fonctionnaire indigène. La traduction de sampagne ${ }^{13}$ par « Sampanjer » reproduit le décalage phonétique que l'on lit dans la version française. Le traducteur est aussi fidèle dans la traduction de «fchfchfchfchfch» par «Fschfschfschfschfsch» où il reproduit, en ajoutant la lettre s entre $f$ et $c$, le même effet phonétique que dans l'original. En revanche, la traduction ne réussit pas à rendre vin d'honneu' de façon à marquer la non-prononciation du $r$ de la fin. Cela est bien évidemment dû au fait que dans « Festwein » il n'y a pas de $r$. Ainsi, la marque du petitnègre ${ }^{14}$ disparaît dans la traduction, non à cause d'une erreur de la part du traducteur, mais du fait de la différence même des langues.

Dans l'exemple suivant, il s'opère en revanche dans la traduction une certaine correction du petit-nègre, bien qu'il eût été possible pour le traducteur de rendre la structure du petit-nègre sans prendre le risque d'opacifier davantage le texte ${ }^{15}$ :

Le garde avala sa salive puis, mettant toute sa rancune dans le regard qu'il jeta à Meka, dit d'un seul trait dans son parler elliptique que « lui - désignant Meka du doigt - pas rien, pas papier, pas éclairage » (VNM : 167).

Der Gefreite schluckte seine Speichel hinunter und legte seinen ganzen Groll in den Blick, mit dem er Meka ansah. Er zeigte mit dem Finger auf ihn und stotterte in seinem komischen Kauderwelsch : „Er, gar nichts, keine Papiere, keine Erklärung“ (DaNM : 178)

18 On observe dans la traduction allemande une désorganisation du rythme et de la syntaxe du texte original. La présente contribution s'intéressant toutefois au nivellement du texte hétérolingue, il n'est pas utile de s'attarder sur les ajouts d'éléments non nécessaires, la non-concordance syntaxique et les déplacements d'éléments. La structure linguistique de la phrase en parler petit-nègre est surtout corrigée par la traduction de pa $^{16}$ par « keine », « pas de ». Aussi, pas rien est corrigé par « gar nichts », « rien du tout ».

$\mathrm{Y}$ en a vérité, sep (VB : 40)

„Das stimmt, Sep“. (FSTO : 38)

19 La formule «y en a », la mauvaise prononciation de chef («sep »), ainsi que l'élision du déterminant devant "vérité ", sont les marqueurs du petit-nègre dans l'original (voir Delafosse 1904 : 165). Dans la traduction, la particule verbale "y en a » disparaît pour faire place à «das, c'est». Et les traducteurs compensent l'absence de l'article en remplaçant le substantif "vérité » par un verbe. Une autre option aurait été, par exemple, de traduire l'énoncé par «Es gibt Wahrheit », ce qui, cependant, aurait donné une tout autre signification. La structure du petit-nègre pose donc ici un problème qu'il est impossible de résoudre de manière définitive. La déviation lexicale du mot chef, "Sep », qui par ailleurs est expliqué en note de bas de page, reste ici le seul élément du petit-nègre qui a été traduit en allemand ${ }^{17}$. 
Movié, s'exclama le garde, Zeuil-de-Panthère cogner comme Gosier-d'Oiseau ! Lui donner moi coup de pied qui en a fait moi comme soufat'soud !... Zeuil y en a pas rire... (VB: 41$)$

„Hör mal, Alter" rief der Wachmann aus, „Pantherauge prügeln wie Vogelkehle! Er mir einen Fußtritt gegeben, der wie Pech und Schwefel brennen ... Bei Pantheraugen gibt's nichts zu lachen“ (FSTO : 39)

Deux formes de tératologie telle que décrite par Henri Meschonnic (1999: 27) sont présentes dans cet extrait. Il y a des ajouts (comme «Hör mal », «écoute ») et de la nonconcordance («Pantherauge», "CEil de panthère » pour Zeuil). Si les traducteurs réussissent à reproduire en allemand l'emploi de formes simples (l'infinitif " prügeln », «brennen » et le participe passé " gegeben $\left.{ }^{18}\right)$, les déviations lexicales du français, quant à elles, n'ont pas pu être imitées par traduction. Les unités lexicales Movié et Zeuil-de-Panthère sont rendues par "Alter ", "mon vieux », et par "Pantherauge ", " Eil de panthère ». Le mot intraduisible soufat'soud que José Endoença Martins propose de transposer tel quel (Martins 2011 : 166), est remplacé dans la traduction allemande par «Pech und Schwefel », " poix et soufre ». Dans la version allemande, la particule verbale $y$ en a et le pronom moi sont escamotés. La proposition relative « der wie Pech und Schwefel brennen » fait trop correcte du fait du placement du verbe conjugué à la fin. Enfin, la particule verbale $y$ en a dans " $y$ en a pas rire... » se noie dans l'expression presque figée « gibt's nichts zu lachen ». Une rétro-traduction de «Bei Pantheraugen gibt's nichts zu lachen » donnerait « Avec Yeux-de-Panthère on ne rit pas », ce qui constitue un décalage par rapport à l'original au niveau sémantique ${ }^{19}$.

\section{Oralité et configuration de la voix}

21 Pour Henri Meschonnic (1982: 15), «il n'y a pas d'univers du discours sans rythme. C'està-dire sans oralité », car «l'oralité c'est la littérature » (1999: 118). Cependant, Meschonnic s'oppose à la conception ethnologique de l'oralité :

[...] identifiée au parlé, opposée de telle sorte à l'écrit qu'elle n'a qu'une définition négative. C'est l'absence d'écriture des littératures orales. L'écriture appartenait au paradigme du civilisé, du logique. L'oralité était du côté de l'archaïque, de l'exotisme, de la mentalité pré-logique. L'absence d'écriture était une infirmité. (Meschonnic 1982: 16)

Cette représentation de l'oralité est pour Meschonnic « un schéma idéologique grossier et simpliste, où la nostalgie tient lieu d'information anthropologique, de rigueur historique, et de poétique» (Meschonnic 1999: 118). À l'inverse, il propose une définition sociologique et poétique de l'oralité comme « le primat du rythme et de la prosodie dans le sémantique, dans certains modes de signifier, écrits ou parlés. L'intégration du discours dans le corps et dans la voix, et du corps et de la voix dans le discours» (Meschonnic 1982 : 18). L'oralité n'oppose donc pas le parlé à l'écrit. Elle peut être présente tant dans le parlé que dans l'écrit : «il y a [en effet] des écritures orales, et des discours parlés sans oralités » (ibid.). L'oralité n'est pas non plus une particularité exclusive des littératures des sociétés dites archaïques. Au contraire elle est à rencontrer dans toutes les littératures. Toutefois, elle " apparaît le mieux dans [1]es textes portés d'abord par une tradition orale, avant d'être écrits : la Bible en hébreu, ou Homère, les textes africains, toute littérature "populaire" » (cité par Cordingley 2014 : 51). Qui plus est, « [1]es Afriques fantômes et les Orients extrêmes ont ramené au monde de l'écrit l'importance 
symbolique de la parole, qui n'est pas sans rapport avec sa revalorisation » (Meschonnic $1982: 18-19)$.

Un des aspects importants de l'oralité est surtout la configuration de la voix, un de ces « infra-signes » qu'émet le corps. La voix, écrit Meschonnic, «peut faire sa syntaxe, sa rythmique, peut faire sa typologie » $(1982: 21)$. Elle se manifeste par le suprasegmental au niveau lexical (accumulation de syllabes, de consonnes et/ou de voyelles), mais aussi par une déstructuration de la syntaxe et de la ponctuation (notamment l'abondance de points de suspension).

Selon Meschonnic, l'oralité est traduisible, puisque «traduire, c'est traduire l'intraduisible » (1999: 397). Le traducteur se devrait ainsi d'« [...] affiner son oreille pour entendre comment les rythmes prosodiques du texte agencent son sens", afin de "récréer cette spécificité rythmique du discours dans l'oralité d'une autre langue» (Cordingley 2014 : 58). En effet, une bonne traduction est, pour Meschonnic, celle qui ne traduit pas ce que dit le texte, mais « celle qui fait ce que fait le texte, non seulement dans sa fonction sociale de représentation (la littérature), mais dans son fonctionnement sémiotique et sémantique » (1999: 85).

\section{Quand la traduction ne fait pas ce que fait le texte}

Dans les traductions allemandes des œuvres de Ferdinand Oyono, les traducteurs donnent l'impression d'avoir plus lu qu'entendu les originaux. Le rythme et la prosodie de la voix telle que configurée par Oyono en reviennent désorganisées, par endroits d'ailleurs supprimés. À quelques rares occasions, comme dans les exemples ci-dessous, la traduction réussit à rendre en allemand la voix du texte français ${ }^{20}$ :

(1) Môooooot! Répondit l'assistance en imitant le bruit sourd des fesses sur le lit de bambou (VNM : 20)

„Moooooot !“ ahmte die ganze Gesellschaft das dumpfe Geräusch eines Hintern auf dem Bambusbett nach (DaNM : 18)

(2)

Ah oui ! Je vois. C'est ... c'est David Ondoua (VNM : 30)

Ach ja! Das ... das ist David Ondoua (DaNM : 29)

(3)

Le Père ... mon Père ... est... est...mort ...bégayait Martin, dans ... dans ... (VB : 29)

„Der Vater ... mein Vater ...ist ...ist ... gestorben“, stammelte Martin, „....in ... in ...“

FTSO : 27)

Comme on le constate, l'onomatopée Môooooot est rendue dans son rythme en allemand, comptant le même nombre de voyelles (6) qu'en français. Le rythme des phrases (2) et (3) est également rendu comme tel en allemand, le texte traduit plaçant les points de suspension au même endroit, et opérant les réitérations telles qu'elles sont opérées dans l'original. Par ailleurs, en dehors de ces quelques exceptions, la traduction opère un nivellement de la voix du texte original et réorganise le rythme et la prosodie par le déplacement ou la suppression des points de suspension et des syllabes réitérées :

(1)

C' pas les papiers ...que le gou... gou... verneur ...m'a dit ...d'ap ...d'ap ... porter ... C'est la médaille ... qui ...qu'il...est venu me donner, bégayait Meka en continuant à se donner des claques. (VNM : 154)

„Der Gou... Gou... Gouverneur hat mir nicht gesagt, ich... ich ... soll... Papiere 
herbringen. Er hat mir doch die Medaille ...ge... gegeben“, stotterte Meka, indem er sich weiterhin abklopfte. (DaNM : 163)

(2)

Si m...moi ... je ...je dit qu'i ... qu'i ...d'vait met' le bila ... c'est pa'c' que com'ça... le Chef des blancs ... y s'rait baissé pour lui épingler la médaille sur... sur ... son bila !... (VNM : 207)

„Wenn ich...ich...ich...sage, daß...daß er hätte den Bila anziehen sollen... so... so... weil dann der Chef der Weißen ... sich hätte bücken müssen, um ihn die Medaille an... an... an seinen Bila... $z u$ heften (DaNM : 221)

Ici, le rythme des phrases traduites ne concorde pas avec celui de l'original. Dans (1), il y a aussi désorganisation du rythme: au lieu de réitérer la particule "her-» dans «herbringen », la traduction choisit plutôt de répéter «ich». L'effet rythmique de la répétition qui...qu'il ... est compensé par une répétition de la particule "ge-» dans "gegeben ». Dans (2), on observe une transformation du style parlé du texte français en style écrit. Les ellipses de sons et/ou de syllabes que l'on rencontre dans qu'i, d'vait, met', pa'c'que, com'ça et s'rait sont systématiquement nivelées dans la traduction, comme si on avait eu, dans l'original, les mots et expressions «qu'il» («daß er»), "mettre» («anziehen »); « devrait» (« hätte ...sollen»); «parce que» (« weil»); « comme ça » (« dann ») et « se serait baissé » (« sich hätte bücken müssen »).

Enfin, là où la configuration de la voix est réalisée à travers une accumulation de consonnes, de voyelles ou de syllabes à l'intérieur de certains graphèmes, créant du suprasegmental, la traduction allemande réduit ou même efface, comme dans (4) et (7) dans les exemples suivants, l'effet prosodique de cette désarticulation graphique :

(1)

Yéééééééée ! Répondirent les trente voix rauques massées dans la case d’Engamba (VNM : 46)

„Yééé!" antworteten an die dreißig rauhe Stimmen (DaNM : 46)

(2)

- Aaaaaaaaaagathaaaaaa!...

C'est ainsi que Binama, comme un muezzin, appela sa femme depuis la case à palabres.

Ououououououiii ! ... répondit-elle. (VNM : 78)

- „Aaaaaagathaaa!“

Auf diese Weise rief Binama, wie ein Muezzin, von der Palaverhütte aus nach seiner Frau.

"Jaaaa!" antwortete sie. (DaNM : 82)

(3)

Yééééééé, répondit l'assistance (VNM : 96)

„Jaaaaa“ riefen alle. (DaNM : 102)

(4)

De Gaulle, j'arrive, cria-t-elle, j'aaaaaariiriiiive ... ! (VNM : 83)

„De Gaulle, ich komme“, schrie sie. „Ich komme“ (DaNM : 88)

(5)

Ouououououbliéééééééé, cria l'assistance (VNM : 139)

„Vergeeesseeen!“ schrie die Versammlung (DaNM : 149).

(6)

Personne, personne, persooooooooooonne !, vociféra l'assistance (VNM : 140)

„Niemand, niemand, niiemaaand!“ (DaNM : 150)

(7)

Noooooooooooon! répondit toute l'assistance, avec plus de force : Non ! (VNM : 187)

„Nein!“ bestätigten alle noch heftiger : „Nein !“ (VaNM : 199) 
(8)

Yééééé é éé é é é é... approuva la foule (VNM : 190)

„Yéééé!"antwortete die Versammlung (DaNM : 203)

(9)

Aaaaaaaaaaakiééééééé ...! s'exclama-t-il (VB : 20)

„Aaaaaakiééééé!“ rief er aus (FSTO : 17)

\section{Conclusion}

Les traductions allemandes des œuvres de Ferdinand Oyono affichent un certain manque de cohérence et de continuité dans leurs façons de résoudre les problèmes que pose l'imaginaire multilingue et hybride de l'écriture de l'auteur camerounais. Elles réussissent, comme nous l'avons vu, à reproduire quelques structures du français petitnègre en allemand (utilisation du $s$ au lieu du ch, emploi de formes verbales simplifiées telles que l'infinitif et le participe passé). Par contre, certaines déviations lexicales et syntaxiques caractéristiques du français petit-nègre demeurent intraduisibles du fait de l'absence, en allemand, de structures similaires. En effet, la question linguistique n'a pas été abordée de la même manière dans les imaginaires coloniaux allemand et français, ce qui a eu pour résultat que dans la langue allemande, il n'existe pas de parlure qu'on pourrait comparer au français petit-nègre. Par ailleurs, certaines disparités entre les textes originaux et les traductions, notamment au niveau de la configuration de la voix, sont le fait de choix poétiques des traducteurs. Cherchant à produire un style plus écrit qu'oral, ils en arrivent, à certains endroits, à écrire une traduction qui dit ce que dit l'original, mais ne fait pas ce que ce dernier fait.

\section{BIBLIOGRAPHIE}

Amedegnato, Oyou Sénamin, 2010, « "Non langue” et littérature. L'exemple du parler petitnègre », dans M. Ngalasso-Mwatha, (dir.) L'Imaginaire linguistique dans les discours littéraires, politiques et médiatiques en Afrique, Pessac, Presses universitaires de Bordeaux, p. 97-114.

Auzas, Noémie, 2009, Chamoiseau ou les voix de Babel. De l'imaginaire des langues, Paris, Imago. Bassnett, Susan et Lefevere, André, 2010, Constructing Cultures. Essays on Literary Translation, Clevedon, Multilingual Matters.

Bedi, Lasme Elvis, 2006, Deutsch in Afrika: Vergangenheit, Gegenwart, Zukunft, Hamburg, Verlag Dr. Kovač.

Biloa, Edmond, 2010, « L'imaginaire linguistique dans le discours littéraire », dans M. NgalassoMwatha (dir.), L'Imaginaire linguistique dans les discours littéraires, politiques et médiatiques en Afrique, Pessac, Presses universitaires de Bordeaux, p. 71-96.

Chevrier, Jacques, 1984, Littérature nègre, nouvelle édition augmentée, Paris, Colin.

Cordingley, Anthony, 2014, « L'oralité selon Henri Meschonnic », Palimpsestes, n² 27, p. 47-60. 
Delafosse, Maurice, 1904, Vocabulaire comparatif de plus de soixante langues ou dialectes parlés à la Côte d'Ivoire, Paris, Leroux.

Glissant, Édouard, 1996, Introduction à une poétique du divers, Paris, Gallimard.

Hassan, Siddi Eyman, 2010, «Écrire dans la langue de l'autre : un dilemme dans une quête d'identité », dans M. Ngalasso-Mwatha, (dir.), L'Imaginaire linguistique dans les discours littéraires, politiques et médiatiques en Afrique, Pessac, Presses universitaires de Bordeaux, p. 115-131. Lennon, Kathleen, 2015, Imagination and the Imaginary, Hoboken, Taylor and Francis.

Kolesch, Doris, 1992, « Schwarze Haut, weisse Sprachen. Zur Dekonstruktion der kolonialen Diskurse in Ferdinand Oyonos Une vie de boy ", Zeitschrift für Literaturwissenschaft und Linguistik, vol. 22, $\mathrm{n}^{\circ} 85$, p. $12-32$.

Martins, José Endoença, 2011, « Pidgins et créoles africains dans la traduction littéraire », Todas as Letras $R, \mathrm{n}^{\circ} 13$ (2), p. 160-168.

Meschonnic, Henri, 1982, «Qu'entendez-vous par oralité ? ", Langue française, n 56, p. 6-23.

Meschonnic, Henri, 1999, Poétique du traduire, Lagrasse, Verdier.

Meschonnic, Henri, 2008, Dans le bois de la langue, Paris, Laurence Teper.

Milton, John et Bandia, Paul Fadio, 2009, « Agents of Translation and Translation Studies ", dans J. Milton et P. F. Bandia (dir.), Agents of Translation, Amsterdam, Philadelphie, John Benjamins, p. 1-18.

Moura, Jean-Marc, [1999] 2013, Littératures francophones et théorie postcoloniale, Paris, PUF.

Ngalasso-Mwatha, Musanji, 2010, « Avant-propos », dans M. Ngalasso-Mwatha (dir.), L'Imaginaire linguistique dans les discours littéraires, politiques et médiatiques en Afrique, Pessac, Presses universitaires de Bordeaux, p. 11-25.

Van den Avenne, Cécile, 2005, «Bambara et français-tirailleur », Documents pour l'histoire du français langue étrangère ou seconde, $\mathrm{n}^{\circ} 35$, [En ligne], https://journals.openedition.org/dhfles/1115 , consulté le 20 janvier 2018.

\section{Corpus}

Oyono, Ferdinand, 1956a, Le Vieux Nègre et la Médaille [VNM], Paris, Julliard.

Oyono, Ferdinand, 1956b, Une vie de boy [VB], Paris, Julliard.

Oyono, Ferdinand, 1957, Der alte Neger und die Medaille [DaNM], trad. Katharina et Heinrich Arndt, Düsseldorf, Progress Verlag Johann Fladung.

Oyono, Ferdinand, 1958, Flüchtige Spur Tundi Ondua [FSTO], trad. Katharina et Heinrich Arndt, Düsseldorf, Progress Verlag Johann Fladung.

\section{NOTES}

1. " gleichberehtigte Verständigungsweise ".

2. Si la notion d'imaginaire désigne, comme l'écrit Kathleen Lennon, l'ensemble des modèles, formes et images sur lesquelles sont basées notre expérience et notre conception du monde (Lennon 2015 : 1), l'imaginaire linguistique est « cette idée selon laquelle les langues naturelles sont des espaces de projection sur l'imaginaire» (Auzas 2009: 11). Chaque langue naturelle 
donnerait de ce fait accès à un réservoir d'images et de modèles de représentation du monde, de soi et des autres, et construirait une certaine Weltanschauung. Mais l'imaginaire linguistique désigne aussi - selon une définition d'Anne-Marie Houdebine-Gravaud - le « rapport du sujet à la langue, à la sienne et celle de la communauté qui l'intègre comme sujet parlant - sujet social ou dans laquelle il désire être intégré, par laquelle il désire être identifié, par et dans sa parole » (citée par Hassan 2010 : 115). Par ailleurs, comme l'écrit Edmond Biloa, « lorsque l'on se situe du point de vue du texte littéraire, il est en fait question du rapport d'un écrivain à la langue/aux langues » (Biloa 2010 : 72). Il peut évidemment aussi s'agir du rapport d'une langue à une autre (ou aux autres) dans la littérature, aussi bien que du rapport des personnages à leur langue ou à celle de l'autre.

3. S'il existe un imaginaire de l'auteur, on peut pareillement parler d'un imaginaire du traducteur. À ce propos, Édouard Glissant écrit que ce que l'auteur réalise « par la pratique de sa langue d'expression » le traducteur le réalise " par le passage d'une langue à une autre, confronté à l'unicité de chacune de ces langues"(Glissant 1996: 45). L'imaginaire du traducteur peut désigner entre autres son rapport à l'original, à la langue et à la culture de l'original, la représentation qu'il a de ceux-ci (du texte étranger, de sa langue et de sa culture), aussi bien que dans sa conception du rôle du traducteur, et du rapport de sa langue à la langue qu'il traduit.

4. "Translation never takes place in a vacuum; it always happens in a continuum, and the context in which the translation takes place necessarily affects how the translation is made. Just as the norms and constraints of the source culture play their part in the creation of the source text, so the norms and conventions of the target culture play their inevitable role in the creation of the translation. »

5. Noémie Auzas parlerait de l'imaginaire d'une langue-narcisse (2009 : 46).

6. Désigné dans la suite de cet article par l'abréviation VNM.

7. Désigné dans la suite du texte par l'abréviation VB.

8. Comme l'écrit Ngalasso-Mwatha, l'imaginaire linguistique des littératures africaines, «se manifeste au travers des images et représentations idéologiques véhiculées par les textes en même temps que par la création de nouvelles formes linguistiques au [sic] niveaux lexical, grammatical, énonciatif et rhétorique » (Ngalasso-Mwatha $2010: 23$ ).

9. Il s'agit du père Vandermayer dans Le Vieux Nègre et la Médaille (« As-tu la maladie du sommeil ? lui-demanda-t-il dans un mauvais mvema » (12); et dans Une vie de boy, du père Gilbert («Il connaissait quelques mots Ndjem, mais il les prononçait si mal qu'il leur donnait un sens obscène » (18)) et de son adjoint, père Vandermayer (« Il les faisait mettre nues dans son bureau, tout en répétant dans un mauvais Ndjem » (27)).

10. «[...] das komplexe Sprachengefüge in den Kolonien, die Schwierigkeiten bezüglich der Sendung von Lehrkräften aus dem Mutterland und die Einstellungen gegenüber den Grundlagen einer allgemeinen Volksbildung. Der letzte Grund scheint das Hauptgewicht zu tragen ».

11. Il existe, certes, des variantes hybrides de l'allemand en Namibie et en Afrique du Sud. Cependant, leur constitution et leur portée ne sauraient être comparées au parler petit-nègre qui naît de l'interaction entre colons et colonisés, officiers et sous-officiers blancs, et qui était répandu dans toutes les colonies françaises d'Afrique.

12. Tous les italiques dans les extraits suivants ont été ajoutés par nos soins.

13. "Sampagne " signale ici le français petit-nègre en ce sens que, d'après les observations de Delafosse, « beaucoup » de locuteurs indigènes « remplacent le ch par un s [...] » (Delafosse 1904 : 265).

14. Selon Maurice Delafosse, les Noirs auraient «au début une grande difficulté à terminer un mot par une consonne [...]» (Delafosse 1904 : 265). C'est ce qu'on observe ici à travers l'élision de la consonne $r$ chez le fonctionnaire indigène.

15. Nous soulignons. 
16. "La négation exprimée seulement par le mot "pas" " est un des indices principaux du petitnègre (Delafosse $1904: 265$; Amedegnato $2010: 102$ ).

17. Nous soulignons.

18. La toute première caractéristique du petit-nègre citée par Delafosse est « l'emploi des verbes à leur forme la plus simple (infinitif pour les verbes de la $1^{\text {re }}$ conjugaison, participe passé ou impératif ou encore infinitif ramené à la $1^{\text {re }}$ conjugaison pour les verbes des $2^{\mathrm{e}} 3^{\mathrm{e}}$ et $4^{\mathrm{e}}$ conjugaisons) » (ibid.).

19. L'original en français standard aurait été : « Yeux ne rit pas ».

20. Nous soulignons.

\section{RÉSUMÉS}

Dans son ouvrage Dans le bois de la langue, Henri Meschonnic souligne la nécessité d'« oublier la langue pour défendre les langues ", clamant que «l'ennemi des langues, c'est la langue ». Lui faisant écho, Glissant développe le concept de l'imaginaire des langues et l'idée d'une écriture multilingue qui met sa langue propre en rapport avec les autres langues du monde. Glissant comme Meschonnic proposent une approche traductrice qui sache rendre compte non seulement de l'hétérolinguisme, mais aussi de l'oralité et du rythme du texte littéraire. Se servant de cette approche, le présent article examine l'imaginaire de la traduction des romans du Camerounais Ferdinand Oyono en allemand. Il se concentre notamment sur l'analyse de la traduction en allemand du parler petit-nègre et de la configuration de la voix dans le texte original. L'objectif est de déterminer si la traduction entend le texte original et fait ce que ce dernier fait.

In his book Dans le bois de la langue, Henri Meschonnic underlines the necessity of "forgetting the language in order to defend languages," claiming that "the enemy of languages is language." In the same vein, Édouard Glissant develops the concept of imaginary of languages and the idea of a multilingual writing which puts the own language in relation to other languages of the world. Glissant, like Meschonnic, proposes a translation approach which should be able to give full account of the heterolingualism as well as of the orality and rhythm of the literary text. Making use of this approach, the present paper examines the imaginary of the German translation of novels from the Cameroonian author Ferdinand Oyono. It focuses on the analysis of the translation of utterances in the petit-nègre register, and of the voice configuration in the original text. It aims to determine whether the translation "listens to" the text and does what the text does.

\section{INDEX}

Mots-clés : traduction, imaginaire, multilinguisme, Oyono (Ferdinand), petit-nègre, oralité, hétérolinguisme

Keywords : translation, imaginary, multilingualism, Oyono (Ferdinand), petit-nègre, orality, heterolingualism 


\section{AUTEUR}

\section{EL-SHADDAI DEVA}

DFG Research Training Group « Globalization and Literature », Ludwigs-Maximilian University Munich 